\title{
Evidence for a fundamental property of steering
}

\author{
Ola Benderius \\ Chalmers University of Technology \\ Göteborg, Sweden
}

\author{
Gustav Markkula \\ Volvo Group Trucks Technology \\ Chalmers University of Technology \\ Göteborg, Sweden
}

\begin{abstract}
In this paper, a general and fundamental property of steering is demonstrated: It is shown that steering corrections generally follow bell-shaped profiles of steering rate. The finding is strongly related to what is already known about reaching movements. Also, a strong linear relationship was found between the maximum steering wheel rate and the steering wheel deflection, something that indicates a constant movement time for the correction. Furthermore, by closer examination of those corrections that cannot be described by a single bell-shaped rate profile, it was found that they typically can be described using two or, in some cases three or four, overlapping profiles, something which relates to superposition of motor primitives.
\end{abstract}

\section{INTRODUCTION}

From studying normal reaching behavior, it is well-known (Morasso, 1981) that the velocity of the hand, rather than the joints, follows a distinct bell-shaped pattern over time, as illustrated in Fig. 1. In other words, it seems the brain directs the hand towards the target, rather than setting the angles of the individual joints (see also Haggard, Hutchinson, \& Stein, 1995). In many of the early studies (e.g Morasso, 1981; Flash \& Hogan, 1985; Haggard et al., 1995; Won \& Hogan, 1995), movement was, by experimental setup, restricted to two dimensions. However, in later studies the same type of behavior have been confirmed in three dimensions as well (Atkeson \& Hollerbach, 1985; Grimme, Lipinski, \& Schöner, 2012).

In this paper, a large amount of driving data from different data sets was used in an effort to investigate the same kind of velocity behavior in the one-dimensional case. Here, the one dimension corresponds to the driver's rotation of the steering wheel, for which angular rate and deflection was studied. Some results indicating the same type of behavior are available from previous driving research: Breuer (1998) and Markkula, Benderius, and Wahde (submitted) showed a linear relationship between the maximum steering wheel rate and deflection, in a double lane change maneuver and an evasive maneuver, respectively. In the work presented here, the analysis will not only cover large maneuvers, but rather the general case. The linear relationship is interesting for two reasons: (1) the time for any (small or large) steering correction will be roughly constant, and (2) the amplitude of a correction seems predetermined, essentially making it openloop. In itself, the idea of constant movement times is not in any way new. It was suggested in some of the already mentioned work, but actually dates back much further (Bryan, 1892).

The distinct bell-shaped velocity profiles observed for reaching tasks have also been used as an argument when
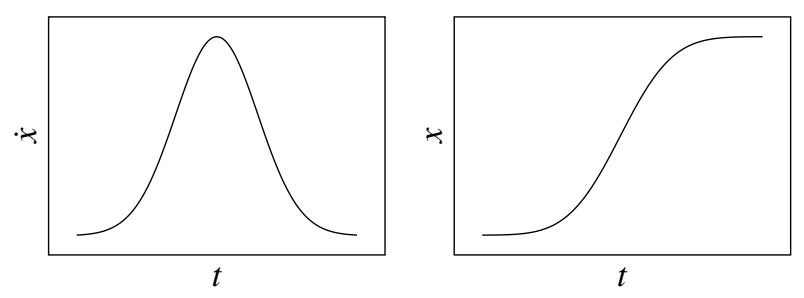

Figure 1. Left: A bell-shaped velocity profile observed, for example, when moving the hand in a reaching task. Right: The distance covered given the same velocity profile.

motivating motor primitives (Bizzi, Mussa-Ivaldi, \& Giszter, 1991; Flash \& Hochner, 2005; Hart \& Giszter, 2010). Here, one such primitive could correspond to a steering wheel correction, either small or large. It has been speculated that all types of movement are composed of such primitives (e.g. Abend, Bizzi, \& Morasso, 1982; Flash \& Hochner, 2005; Bizzi, Cheung, d'Avella, Saltiel, \& Tresch, 2008), and that more complex movements are built from a combination of two or more primitives.

This paper will discuss the following questions:

1. Can steering corrections generally be explained by bell-shaped rate profiles?

2. Is there a linear relationship between the steering wheel rate and deflection, reflecting a constant movement time?

3. Is there any indication of complex steering consisting of several superpositioned motor primitives?

\section{METHOD}

For all the analyses presented below, only the steering wheel angle $\delta(t)$ and the steering wheel rate $\dot{\delta}(t)$, as exem- 


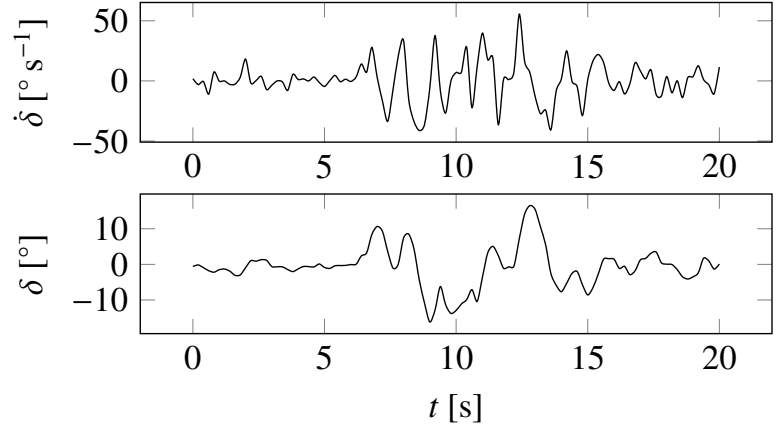

Figure 2. An example of steering wheel rate, and the resulting steering wheel angle.

\begin{tabular}{lrl}
\hline constant & value & unit \\
\hline$\dot{\delta}_{\text {move }}$ & 3 & ${ }^{\circ} \mathrm{s}^{-1}$ \\
$\dot{\delta}_{\text {still }}$ & 1 & ${ }^{\circ} \mathrm{s}^{-1}$ \\
$\Delta \delta_{\min }$ & 2 & $\circ$ \\
$\delta_{\max }$ & 100 & $\circ$ \\
$R_{\text {fit }}^{2}$ & 0.8 & \\
\hline
\end{tabular}

Table 1

Constants used here for extracting steering corrections, and for determining whether or not they are bell-shaped.

plified in Fig. 2, were used. Individual steering corrections were extracted from various data sets.

\section{Identifying corrections}

The steering corrections were extracted chronologically by first detecting steering wheel movement defined by the inequality $|\dot{\delta}(t)|>\dot{\delta}_{\text {move }}$, and from there tracking backwards and forward to find the start $t_{0}$ and end $t_{1}$ of the correction, according to $|\dot{\delta}(t)|>\dot{\delta}_{\text {still }}$. The maximum steering rate was then simply $\dot{\delta}_{m}=\max \left(\left\{\dot{\delta}(t): t \in\left[t_{0}, t_{1}\right]\right\}\right)$.

However, by considering the overall steering wheel deflection $\Delta \delta=\left|\delta\left(t_{1}\right)-\delta\left(t_{0}\right)\right|$, a correction was removed from consideration if (1) the deflection was too small $(\Delta \delta<$ $\left.\Delta \delta_{\min }\right)$, or (2) one or both end-point angles was too large $\left(\max \left(\delta\left(t_{0}\right), \delta\left(t_{1}\right)\right)>\delta_{\max }\right)$. The first constraint was to avoid small steering disturbances not originating from the driver, but rather the vehicle and the road. The second constraint was to focus the analysis on corrections during normal driving, by excluding very large maneuvers. The constants used for the correction extraction are presented in Table 1.

\section{Data}

Data from several sources were used in this work. Each data set will briefly be described below, and a summary is presented in Table 2. Data collected from the driving simulators was logged at 50-100 Hz, while data from the other

\begin{tabular}{ccccccc}
\hline set & subj. & veh. & $\begin{array}{c}\text { s.r. } \\
{[-]}\end{array}$ & $\begin{array}{c}\text { dur. } \\
{[\mathrm{h}]}\end{array}$ & corr. & $\begin{array}{c}f_{c} \\
{\left[\mathrm{~min}^{-1}\right]}\end{array}$ \\
\hline 1 & 20 & $\mathrm{c}$ & 16.2 & 6.3 & 2,946 & 7.80 \\
2 & 12 & $\mathrm{c}$ & 16.4 & 14 & 8,571 & 10.38 \\
3 & 48 & $\mathrm{t}$ & 22.0 & 6.1 & 15,912 & 43.75 \\
4 & 56 & $\mathrm{c}$ & 14.7 & 0.41 & 584 & 23.81 \\
5 & - & $\mathrm{c}$ & $16.0 / 16.4$ & 500 & $3.24 \cdot 10^{5}$ & 10.81 \\
6 & - & $\mathrm{t}$ & 20.0 & 500 & $9.53 \cdot 10^{5}$ & 31.74 \\
\hline
\end{tabular}

Table 2

The data used in this work. The second column shows the number of subjects used, the third the vehicle type (car or truck), the fourth the steering ratio, the fifth the duration of the data, the sixth the number of identified corrections, and the last the number of identified corrections per minute. For the FOT data, the number of subjects was not known.

settings was logged at $10 \mathrm{~Hz}$. The steering wheel diameters were, for passenger cars $38 \mathrm{~cm}$, and trucks $45 \mathrm{~cm}$.

Sets 1 to 3: Driving simulator. The simulator data used were collected in three different studies, of which only the third has been mentioned in other publications so far. All studies had different purposes related to active safety or driver behavior, and were originally not intended for the analysis presented here. Nevertheless, it is believed that the nature of this work is general, and not dependent on any specific data properties or purpose.

In the first study, subjects were asked to drive a passenger car while carrying out repeated distraction tasks. The task was designed so that the subject had to keep a straight course while looking away from the road. At some pre-defined occasions, the subjects car was automatically forced out of the lane and into a head-on collision scenario. Therefore, the data also contain some evasive maneuvers.

In the second study, passenger car drivers were asked to drive four times each, two times under normal conditions, and two times under sleep deprivation. Here, only driving from the normal conditions were used, except for the minor result presented in a later section.

In the third study (Markkula, Benderius, Wolff, \& Wahde, 2013), truck drivers were asked to drive a rigid truck on a highway. The purpose of the study was to test driver behavior when using an electronic stability control (ESC) system installed in half of the drivers' trucks.

The drivers were instructed to drive at a speed of $90 \mathrm{~km} / \mathrm{h}$ in the first study, and at $80 \mathrm{~km} / \mathrm{h}$ in the second and third.

Set 4: Test track. In a test track study, the results of which are not yet published, subjects were instructed to drive on a closed track, and occasionally carry out the same distraction task as in the first study (set 1). The subjects were asked to drive at $70 \mathrm{~km} / \mathrm{h}$, but to slow down in curves.

Sets 5 to 6: Field operational test (FOT). For the purpose of this work, large amounts of steering wheel data were 


\begin{tabular}{cccc}
\hline set & pred. [\%] & $\dot{\delta}_{m}\left[{ }^{\circ} \mathrm{s}^{-1}\right]$ & $\sigma$ \\
\hline 1 & 65 & $22.78(25.67)$ & $0.10(0.04)$ \\
2 & 71 & $16.89(10.59)$ & $0.08(0.03)$ \\
3 & 73 & $19.79(10.97)$ & $0.10(0.04)$ \\
4 & 60 & $20.08(11.74)$ & $0.10(0.05)$ \\
5 & 60 & $13.13(8.50)$ & $0.12(0.05)$ \\
6 & 72 & $16.07(8.62)$ & $0.12(0.04)$ \\
\hline
\end{tabular}

Table 3

The results of fitting each steering wheel rate profile to a single Gaussian function. The prediction rate is the portion of the $R^{2}$ values above the threshold $R_{\mathrm{fit}}^{2}$. See the text for more information.

extracted from FOT data (euroFOT project, 2012), both from passenger cars (set 5) and trucks (set 6). In order to limit data extraction to normal driving, a vehicle speed threshold of $50 \mathrm{~km} / \mathrm{h}$ were used.

\section{Test of steering rate profiles}

In order to determine whether the rate of a steering correction can be considered bell-shaped, each steering rate profile was compared to an ordinary Gaussian function defined as

$$
\dot{\delta}(t)=\dot{\delta}_{m} \exp \left(-\frac{(t-b)^{2}}{2 \sigma^{2}}\right)
$$

where the constant parameters $\dot{\delta}_{m}, b$, and $\sigma$ were obtained through data fitting. If the function, for a given set of parameter values, could fit the data with a coefficient of determination $R^{2}$ above a certain value $R_{\text {fit }}^{2}$, see Table 1 , the correction rate was considered bell-shaped. Important to note is that the standard deviation $\sigma$ is closely coupled with the time duration of the steering correction.

\section{RESULTS}

The results presented here are divided into four sections, three answering the questions outlined in the Introduction, and one for other observations. Throughout, results will be presented separately for each of the six analyzed data sets.

\section{Bell-shaped steering rate}

When fitting a Gaussian function, see Eq. 1 and Fig. 3, to each steering correction in every data set, it was found, as indicated in Table 3, that most corrections in fact could be described by such a function. In the table, the second column shows the portion of all corrections that can be modelled with $R^{2}>R_{\mathrm{fit}}^{2}$, and the third and fourth show the mean and standard deviation for two of the three parameters in Eq. 1. The presented parameters only include cases where the correction was found to be bell-shaped.
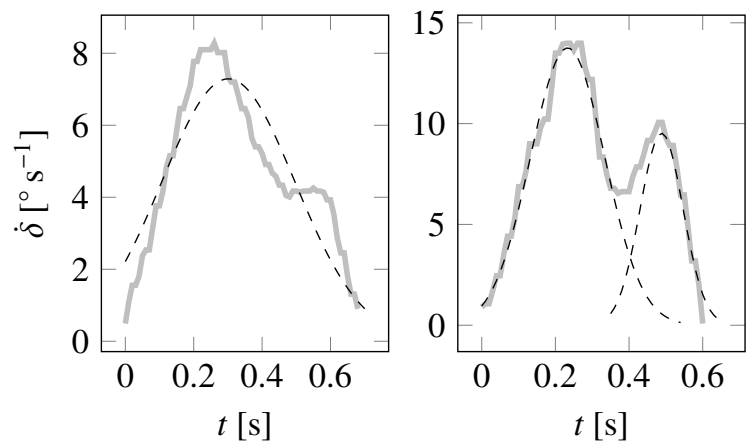

Figure 3. Examples of fitting one (left) and several (right) Gaussian functions to two different steering rate profiles. In the first example $R^{2} \approx R_{\text {fit }}^{2}$.

\begin{tabular}{cccc}
\hline set & $k$ & $R^{2}$ & $\Delta t[\mathrm{~s}]$ \\
\hline 1 & 3.18 & 0.90 & $0.42(0.17)$ \\
2 & 3.80 & 0.86 & $0.38(0.12)$ \\
3 & 3.40 & 0.92 & $0.46(0.16)$ \\
4 & 2.35 & 0.72 & $0.44(0.22)$ \\
5 & 2.92 & 0.81 & $0.54(0.22)$ \\
6 & 3.10 & 0.90 & $0.57(0.19)$ \\
\hline
\end{tabular}

Table 4

The linear relationship between steering wheel rate and deflection for single-Gaussian corrections, where $k$ is the slope, and $R^{2}$ the coefficient of determination.

\section{Constant movement time}

From Table 3 one can note that the parameter $\sigma$ seems fairly constant within data sets, suggesting that the width (time) of the rate profile is rather constant. That is also confirmed in Table 4, where the actual movement time $\Delta t=$ $t_{1}-t_{0}$ was found to be rather constant as well. The distributions of steering correction durations are presented in Fig. 4. Note that only corrections confirmed to be bell-shaped were used in this analysis. Corrections consisting of two or more motor primitives were not expected to be of the same time duration.

The fairly constant average duration of steering corrections, independent of correction size, suggests a relationship between steering wheel rate and deflection. This relation was analysed further and a strong linear relationship was found, as presented in Table 4. From the table, note that the $k$ values are similar, suggesting a similar relation between rate and deflection even between data sets. In Fig. 5, an example of the linear relationship can be found.

\section{Superposition of motor primitives}

As shown above, most steering corrections can accurately be described using a single Gaussian function. For correc- 

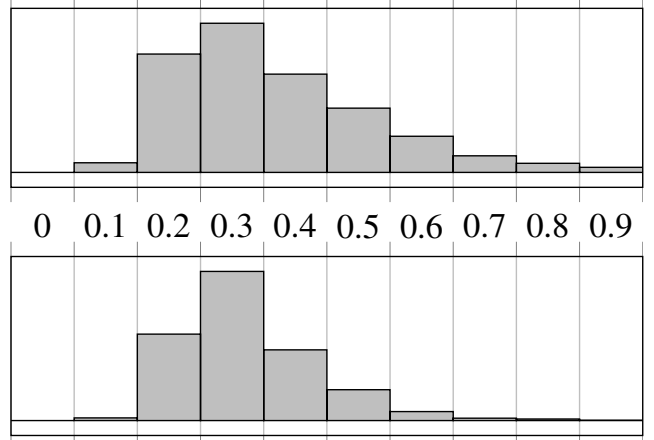

$\begin{array}{llllllllll}0 & 0.1 & 0.2 & 0.3 & 0.4 & 0.5 & 0.6 & 0.7 & 0.8 & 0.9\end{array}$

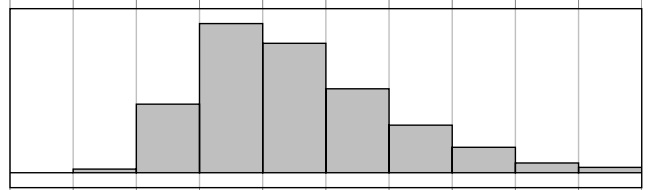

$\begin{array}{llllllllll}0 & 0.1 & 0.2 & 0.3 & 0.4 & 0.5 & 0.6 & 0.7 & 0.8 & 0.9\end{array}$

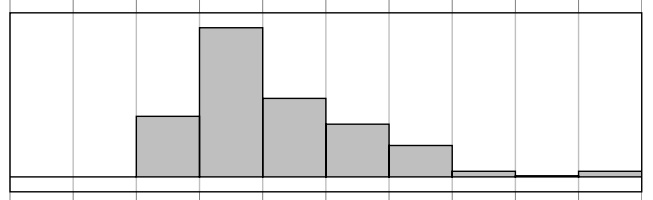

$\begin{array}{llllllllll}0 & 0.1 & 0.2 & 0.3 & 0.4 & 0.5 & 0.6 & 0.7 & 0.8 & 0.9\end{array}$

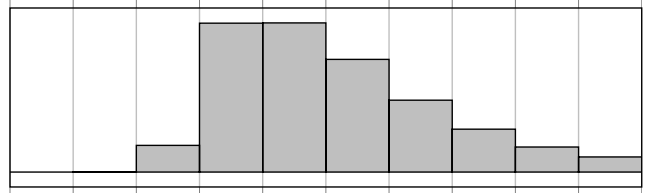

$\begin{array}{lllllllllll}0 & 0.1 & 0.2 & 0.3 & 0.4 & 0.5 & 0.6 & 0.7 & 0.8 & 0.9\end{array}$

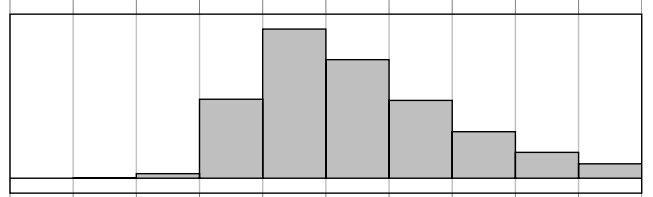

$\begin{array}{llllllllll}0 & 0.1 & 0.2 & 0.3 & 0.4 & 0.5 & 0.6 & 0.7 & 0.8 & 0.9\end{array}$ $t[\mathrm{~s}]$

Figure 4. For each data set, the distribution of durations of the single-Gaussian steering corrections. See also Table 4.

tions that could not be sufficiently described it was found, as shown in Table 5, that the majority could be described using two, or at most four, overlapping Gaussian functions. The same criterion, namely $R^{2}>R_{\text {fit }}^{2}$, was used when determining a good fit, but in this case additional Gaussian functions were added, one at a time, until the criterion was met.

\section{Other}

Three additional analyses were made, in order to investigate differences in steering correction behavior between (1) young and old drivers, (2) male and female drivers, and
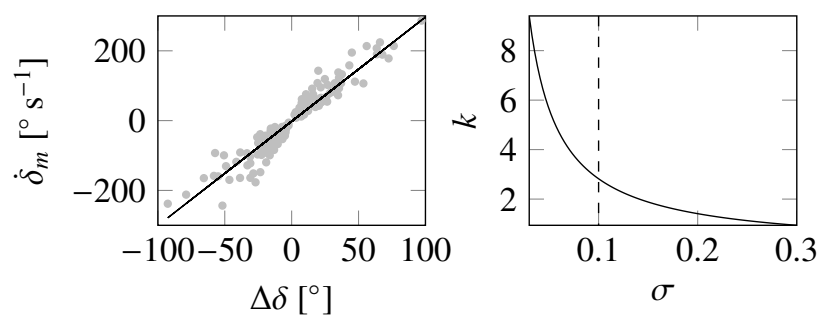

Figure 5. Left: An example (set 1) of the linear relationship between the maximum steering wheel rate and deflection for single-Gaussian corrections. Right: The analytic relation between the standard deviation $\sigma$ and the slope $k$.

\begin{tabular}{ccccc}
\hline set & $1[\%]$ & $2[\%]$ & $3[\%]$ & $4[\%]$ \\
\hline 1 & 65 & 30 & 4 & 1 \\
2 & 71 & 26 & 2 & 0 \\
3 & 73 & 24 & 2 & 0 \\
4 & 60 & 30 & 6 & 3 \\
5 & 60 & 33 & 5 & 1 \\
6 & 72 & 25 & 3 & 0 \\
\hline
\end{tabular}

Table 5

For each data set, the number of motor primitives needed to describe the steering corrections.

(3) alert and sleep deprived drivers. No conclusive differences were found.

\section{DISCUSSION}

The main findings of this work are that the rate of steering wheel corrections generally can be considered bell-shaped, and that steering corrections are of constant duration, thus affirmatively answering the first two questions listed in the Introduction. As for the third question, it has been demonstrated that most steering corrections $(60-70 \%)$ can be described using a single motor primitive. Moreover, the remaining steering corrections generally require only two such primitives.

As a consequence of the constant correction duration and profile shape, there is a strong relationship between steering wheel rate and deflection. Furthermore, the slope coefficient $k$ seems to be rather constant between different data sets, see Table 4.

Based on the data and observations alone, it might be hard to interpret the value of $k$. However, by assuming a Gaussian steering correction, as given by Eq. 1, the relationship can be analytically obtained as

$$
\Delta \delta=\int \dot{\delta}(t) \mathrm{d} t=2 \dot{\delta}_{m} \sigma \sqrt{\pi}
$$

where $\dot{\delta}_{m}$ is the maximum rate, and $\sigma$ the standard deviation 
of the Gaussian function. Then, by noting the linear relationship, $k$ is defined as

$$
\begin{aligned}
\dot{\delta}_{m} & =\frac{\Delta \delta}{2 \sigma \sqrt{\pi}}=k \Delta \delta \\
k & =\frac{1}{2 \sigma \sqrt{\pi}} .
\end{aligned}
$$

By inserting the mean standard deviation $\sigma$ from Table 3, one gets

$$
\sigma=0.1 \rightarrow k=2.8
$$

which is in the same region as the $k$ values presented in Table 4 .

Apart from the central questions of this work, there are some aspects of the results that are interesting to highlight. First, from Table 2 it seems that truck drivers make several times more steering corrections compared to what was observed for the passenger car drivers. For example, this might be explained by the higher steering ratio of the trucks, perhaps since more small corrections are needed to achieve the same total steering compared to passenger cars. Or, it could simply be an effect of the specific method used for extracting corrections, possibly excluding small corrections carried out by the passenger car drivers. There might, of course, also be differences in driving style between the two driver types.

Another interesting observation is the notable differences, between data collected at the test track (set 4), and data collected from passenger cars in the other settings (sets 1, 2, and 5). In particular the number of corrections per minute, but also the number of primitives needed to describe the corrections (see Tables 2 and 5). It might be that the amount of test track data is too small. Another explanation could be that the test track drivers drove on a closed track with relatively demanding curves, which might explain why the modelling of corrections to a larger extent requires more primitives as such driving might require more complex steering behavior.

\section{CONCLUSION}

In this paper, it is shown that steering corrections generally can be explained by bell-shaped rate profiles. For such corrections, a constant movement duration was shown, reflected by a strong relationship between the maximum steering wheel rate and deflection. Furthermore, by closer examination of corrections that could not be described by a single bell-shaped rate profile, it was found that they typically could be described by two, or at most four, motor primitives.

The results of this paper point to a general and fundamental property of steering. Most likely, because of similarities to reaching, this property is not restricted to driving, but has more general validity.

For driver modelling, the results might be of particular importance. Traditionally, steering is modelled as a purely closed-loop continuous control action. Here, it was indicated that steering might rather be composed of intermittent openloop control bursts. Markkula (2014) has incorporated this aspect of control into a more general framework for driver control behavior modelling.

\section{References}

Abend, W., Bizzi, E., \& Morasso, P. (1982). Human arm trajectory formation. Brain: a journal of neurology, 105(Pt 2), 331-348.

Atkeson, C. G., \& Hollerbach, J. M. (1985). Kinematic features of unrestrained vertical arm movements. The Journal of Neuroscience, 5(9), 2318-2330.

Bizzi, E., Cheung, V., d'Avella, A., Saltiel, P., \& Tresch, M. (2008). Combining modules for movement. Brain Research Reviews, 57(1), 125-133.

Bizzi, E., Mussa-Ivaldi, F. A., \& Giszter, S. (1991). Computations underlying the execution of movement: a biological perspective. Science, 253(5017), 287-291.

Breuer, J. J. (1998). Analysis of driver-vehicle-interactions in an evasive manoueuvre-results of moose test studies. In Proceedings of the international technical conference on enhanced safety in vehicles.

Bryan, W. L. (1892). On the development of voluntary motor ability. The American Journal of Psychology, 125-204.

euroFOT project. (2012). http://www.eurofot-ip.eu. (Accessed: 2014-02-20)

Flash, T., \& Hochner, B. (2005). Motor primitives in vertebrates and invertebrates. Current opinion in neurobiology, 15(6), 660666.

Flash, T., \& Hogan, N. (1985). The coordination of arm movements: an experimentally confirmed mathematical model. The Journal of Neuroscience, 5(7), 1688-1703.

Grimme, B., Lipinski, J., \& Schöner, G. (2012). Naturalistic arm movements during obstacle avoidance in $3 \mathrm{~d}$ and the identification of movement primitives. Experimental Brain Research, 222(3), 185-200.

Haggard, P., Hutchinson, K., \& Stein, J. (1995). Patterns of coordinated multi-joint movement. Experimental Brain Research, 107(2), 254-266.

Hart, C. B., \& Giszter, S. F. (2010). A neural basis for motor primitives in the spinal cord. The Journal of Neuroscience, 30(4), $1322-1336$.

Markkula, G. (2014). Modeling driver control behavior in both routine and near-accident driving. In Proceedings of the human factors and ergonomics society 58th annual meeting.

Markkula, G., Benderius, O., \& Wahde, M. (submitted). Comparing and validating models of driver steering behaviour in collision avoidance and vehicle stabilization. Vehicle System Dynamics.

Markkula, G., Benderius, O., Wolff, K., \& Wahde, M. (2013). Effects of experience and electronic stability control on low friction collision avoidance in a truck driving simulator. Accident Analysis $\mathcal{G}$ Prevention, 50, 1266-1277.

Morasso, P. (1981). Spatial control of arm movements. Experimental Brain Research, 42(2), 223-227.

Won, J., \& Hogan, N. (1995). Stability properties of human reaching movements. Experimental Brain Research, 107(1), 125136. 